Die chemische Zusammensetzung des Fisehbeins hat die grörste Aehnlichkeit mit ler des Horns, unterscheiciet sich jedoch daron durch die Gregenwart der Gallerte und darch eine größsere Menge fetter Substanz, welchen beiden Körpern dasselbe ohne $Z$ weifel seine grülsere Biegsamleit verdanlit.

Die chemische Zuswmensetzung der Menschentaare, die des Horns der hräuterfressenden Thiere und der larten der Fische ist beinahe identisch, obgleich das Element, wo. rin diese Thiere leben und ihre Nahrungsmittel sehr verschiedener Natur sind.

Zweite Abtheilung.

Pharmaceutische Naturgeschichte und Pharmokognosic.

\title{
Ueber Radix Jalapae und ihre Mutterpflanze
} von

Nees v. Esenbeck u. Cl. Marquart.

5. $x$.

Seitdem Herr Dr. Schiede in Mexico und zwar in der Gegend ron Xalapa eine neue Art der Gattung Ipomoca enideckte, deren Wurzeln als Arzneimittel eingesammelt werden, sind unsere Pharmakologen riemlich alkgemein der Meinung, dafs diese Pflanze und nicht die früher dafür angenommene I. Jalapa Desf. als die Nutterwurzel unserer offi. cinellen Jalapen-Wurzel zu betrachten sey, obne dafs uns bis jetzi eine genaue Prüfung dieses gewifs nicht unwichtigen Gegenstandes belannt geworden ist. Jene neue Art der 
Gatlung Ipomoea ist unter dem Namen I. Purga ron Herrn Prof. Wenderoth zuerst beschrieben und von N. v. E. in seinen Supplementen der' Sammlung officineller Pflanzen zuerst abgebildet worden. Sie hat sich indessen durch die meisten botanischen Gürten verbreitet und unter andern auch in Jem hirsigen bot. Garten in vergangenen Jerbste sehr reichlicb im freien Lande geblïht. Ueber ihre Verschiedenbeit als eigene neue Art ist laher wohl bei uns kein $Z$ weí fol mehr; auch gehört sicher die von Nuttạl als Ip. Jalapa beschriebene Pflanze hierher. ${ }^{*}$ ) Dieser Name kann aber nicht angenommen werden, da er stets zu Verwechslungen mit L Jalapa Desf. (Cant. Jalapa Autor.) tïhren würde. -Unter den ältern Autoren möchten wir annchmen, dafs die Mechoacanna vera sativa des Munting (Naubeurige Beschreywing der Aardgewassen) als Synonym ebenfalls hiarher zu ziehen sey, um so mehr, da wir jener Pllanze, seitdem wir die Mirabilis longiflora als Muttespflanze der Rad. Mechoacannae griseac kennen, eine Wurzel mit ähnlicher Wirksamkeit zuschreiben dürfen. Munting verstand wahrscheinlich unter seiner Mechoacanna vera dic stärkere Rad. Jalapac und unter Mechoacanna sylvestris die schwächere Rad. Mechoacanua grisea.

$$
\text { 6. } 2 .
$$

Durch die folgende Untersuchung hoffen wir nun die wirkliche I Hentität der Rad. Ipomeae Purgae mit der officinellen Bad. Jalapae darzuthun. N. ז. E. batte nämlich die Freude, in diesem Herbste bei seinem verehrten Freunde, Uerrn Med. Assessor Dr. Wild in Kassel, der die Ipomoea Purga zuerst mit Erfolg cultivirte, eine blsine Quantilät der getrockneten Wurzeln dieser Pflanze au sehen and van,demselben einige Stücke zum Geschonk au erbalten. Diese ge-

7) Centralblatt 1833 p. 790. 
troekneten Wurceln hatten ganz das Ansehen der ächten Jalapa; die Stücke sind theils ganz, birnförmig, theils halbirt oder dreiseitig; die äufsere mit der Rinde bedechte Seite ist sehr stark runzlich, ron duntler graubranner Farbe, im Innern ist die Textur sebr dicht, feinfaserig; die Farbe ist schmutzig weifs mit dunklera Stellen gemischt. Das specif. Gew. der Wurzel ist ungefähr das der officinellen Jalapa, der Geruch ist der letztern ähnlich, doch schwächer; der Gieschmach anfangs unmerklich, mehlig, später anhaitend Hratzend.

\section{3.}

Bevor wir nun zu der chemischen Prüfung and Vergleichung übergehen, sey es uns erlaubt, hier einer falschen Jalapewurzel zu erwähnen, von der wir durch die Güte unsres verehrten Freundes, des Herrn Prof. Geiger, schon vor geraumer Zeit eine Probe erhielten und die, wio uns ein Droguist aus hiesiger Gegend versichert, auch bei uns unter der gewöhnlichen Rad. Jalapae vorkommt und für eine gesingere Sorte dersellen gilt. Diese Wurzel kommt theils in grölsern, inuen flachen aulsen gewölbten, theils auch in heinern walzenförmigen oder gekrümmten fingerdichen Stükken vor. Unsere grölsere Stücke sind an drei Zoll lang, 2. Zoll breit, 5-10 Linien dick; sie zeigen auf der Rindenseite starke Längsfurchen, die Farbe ist hier blafsbraun mit duribiern Flecken, etwas heller als die der äcbten Jalapa; die innere Seite zeichnet sich durch starke Fasern, die man hie und da bemerkt, aus. Der Querschnitt zeigt eine dichte T'extur, eine blafsgraue Farbe mit dunllern Stellen gemischt und schmale koncentrische Linien; mit der Joupe betrachtet bemerkt man hleine glänzende weifse Punkte, die ohne $Z_{\text {weifel }}$ ron einem Salze, wie bei der Rad. Mechoacannae, herrühren; doch sind diese Punkte weit kleiner und in geringerer Menge als bei der Mechoncanna 
vorhanden. Der Geruch ist ebenfalls etwas der Jalapenwarzel ähnlich, doch viel schwächer; der Geschmack ist derselbe. Die kleinen Stücke sind leichter unj gewifs minder reich an Harz. Mit der zuerst ron S chwe insberg beschriebenen falschen Jajape ist die unsrige nicht za verwechseln, eben so wenig mit Rad. Mechoacannae. Die fingerdichen Stüche ha. ben dagegen einige Aehnlichleit mit Turpithwarzel.

\section{4 .}

Ueber die Abstammung dieser falschen Jalape können wir zwar nichts mit voller Sicherbeit bestimmen; da wir aber hören, dafs diese Wurzel unter der gewöhnlichen Jalape vorhommt, so dürfen wir annehmen, dals sie gleiches Vaterland mit ihr habe. Berücksichtigen wir nun zugleich die grüfseren Stücke, welche nur von einer bedeutend starken Wurzel abstammen können, so dürfen wir mit ziernlicher Wahrscheinlichkeit annehmen, dafs diese falsche Jalapenwurzel der Ipomoea Jalapa Desf. angehöre, dic belanntlich in dera wärmern Theile ron Mexiko vorkommt and deren Wur zel oft eine Schwere von 10 bis 12 Pfund erlangt. *) In dieser bedeutenden Gröfse der Wurzel liegt zugleich ein Grund mehr für die jetzige Annahme, dafs unsere officinelle Jalapenwurzel, die nie in bedeutend grofsen Segmenten rorkam, nicht von dieser Ipom. Jalapa Desf., wie man bisher glaubte, gesanmelt weade. - Uebrigens bestätigt uns das sehr äbnliche chemische Verhalten der beiden hier beschriebenen Wurzeln die Annahme, dafs beide einer Pllanzengattung angehören. Für die Pharmacie ist diese falsche Jalape (wenn sie diesen Namen, womit wir sie zum Unterschiede bezeichnen, überhaupt verdient?) wegen des unten erwähnten käuflichen Harzes sehr wichtig.

*) S. die Abbild. in N. v. E. Arzneipflanzen. 


\author{
6. 5.
}

Zur chemischen Prüfung und Vergleichung wurden jetzr fulgende Versuche angestellt: 120 Gran gröblich zerkleinerte Wurzeln der Ipomoea Purga wurden in gelinder Wärme mit 3 Unzen Alkohol von 85 Proc. digerirt, die Flüssigheit nach einigen Tagen abgegossen und dieselbe Operation wiederholt, während die vollständige Erschöpfung mit schwächerem Weingeiste ausgeführt wurde. Die ersten Tinkturen waren schwach gelblich gefärbt; die letztere, mit schwächerm Weingeiste, hellbraun. Die vereinigten Flïssigkeiten wurden klar filtrirt und zur Trockne abgeraucht. Der Rückstand mit destillirtem Wasser behandelt, trennte sich in 2 wei Theile; die Har filtrirte wăssrige Lösung hinterliełs $24^{1 / 2}$ Gran Extraltivstoff und das in einem Uhrgläschen gesammelte Harz wog $14^{\frac{1}{2}}$ Gran.

\title{
6. 6.
}

Das erhaltene Harz war von gelber Bernsținfarbe, durchsichtig tnd schmechte kratzend, roch erwürmt dem Jalapenharze ähnlich, aber schwächer. Es löste sich micht in Terpenthinül, färbte den Schwefëäther schwach und lëste sich leitht in Weingeist, Aetzhaillauge und rauchender Salpetersäure; in den beiden letzten Füssigkeiten mit dunbler Farbe. Die weingeistige Lüsung wurde durch Aetzkalilauge nur dunkler gefärbt, nicht gefällt. Die alkalische Lüsung nit Wasser verdünnt und mit koncentrirter Schwefelsäure in Ueberschufs versetzt, wurde milchig; nach einiger Ruhe trennten sich weirse Flocken, welche sich an der Oberfläche der FlüssigHeit sammelten.

\section{7.}

Der Extraktivstoff aus der Wurzel der Ipomoea Purga war dunkelbraun, hygroskopisch und löste sich leicht in Wasser und Weingeist; er war fast ohne Geruch und schmechte nicht unangenehn, sëuerlich süfs, hintennaob haum etwas 
kratzend. Die wïssrige Lösung röthete das Lakmuspapier stark; scherefelsaures Eisenoxydul bewirbte in derselben anfangs keine Veränderung, später einen kaffeebrounen Niederschlag, *) Eisenchlorid dunkel olivengrüne Färbang; Queck sitherchlorid sehr geringen bla?s gelblich braunen Niederschlag, nautrales essigsaures Blei und basisch essigsaures Blevoxyd dagegen sehir reichliche schmutzig hellgelbliche Niederschlngge und Entfärburng der Flüssigheit; salpetersaures Silber brachte einen reichlicben schwarzgrauen Niederschlag, Hlecsaurcs Ammonium aber heine Veränderung hervor; Zinnchlorür gab einen gelblich weifsen reichlichen Niederschlag and Leimlösang reichliche flockige Ausscheidung.

$$
\text { 6. } 8 .
$$

120 Gr. Jalapenwurzel der Otficinen wurden auf gleiche Weise bebandelt; die Tinlturen waren sämmtlich dunliler hellbraun als die der Ipomoea Purga und lieferten 33 Gran Extraktirstoff und 16 Gran Harz. Das Harz hatte das gewöhnliche Ansehen, wie es in den Apothelien vorkommt, löste sich nicht in Terpenthinöl, färbte den Schwefeläther hanm merklich und verhielt sich gegen Weingeist, Aetzhalilauge und Salpetersäure wie das Harz der Purga. Die Lösung in Weingeist von 85 Proc. wurde, gegen die Benbachtung von $\mathrm{C}$ a det und $\mathrm{Gerber}$, weder durch einzelne Tro. pfen, noch durch Vermischung mit gleichen Theilen starker Aetzkalilauge gefällt, sondern blicb hlar end färkte sich. dunller. Die alkalische Lösung mit Wasser verdünnt und mit Koncentrirter Schwefelsäure übersättigt verhiclt sich eben 80 wie die des Purgaharzes, nur war die Ausscheidung dunklex.

*) Alle Erscheinungen, wenn es nicht ausdrücklich anders bemerkt ist, wurden 24 Stundeu nach den Zusatze der Reagen. tien beobachiet und beschrichen 


\section{9.}

Der Extrahtivstoft war in seinen phrsischen Eigenschaften von dem Extraktirstoffe der Ip. Purga nicht zu unterscheiden. Seine wässrige Lösung röthete ebenfalls das Lakmuspapier stark, ward voun schwefelsauren Eisenoxydul anfangs nicht verändert, später trat eine starke olivenfarbene Trübung ein, während sie vom Eisenchlorid braungrün gefärbt wurde. Durch Quechsilberchlorid entstand eine schwache Trübung und neutrales essigsaures Blei fällte sie blafs schmutzig gelblich braun, mit oben stehender gelber Flüssigkeit; $b a$ sisch essigsaures Blei aber brachte einen Niederschlag von blasserer, etwas röthlicher Farbe und Entfärbung der Flüssigkeit hervor, salpetersaures Silber schlug sie reichlich schwarzgrau nieder; von kleasaurem Ammonium ward sie kaum getrübt; Zinnchlorïr wirkte wie basisch essigsaures Blei und Leimlösung brachte heine Veränderung herror.

9. 10.

Endlicb wurden auch $120 \mathrm{Gr}$. der oben beschriebenen Jalapa spuria mit Weingeist auf angegebene Weise extrahirt und dadurch 22 Gran Harz and 8 Gran Extraktivstoff erhalten. Das Harz war im äufsern Ansehen und im Geschmacke nicht von dem Jalapenharze zu unterscheiden; der Geruch schien etwas schwächer, aber sein Verbalten gegen die Lö. sungsmittel war ganz verschieden. Terpenthinöl löste über die Hälfte; Weingeist und Schwefeläther lösten es völlig auf; eben so Aetzhalilauge und Salpetersäure. Die weingeistige Lösung verhielt sich gegen Aetzhalilauge wie die des oben geprüften Jalapenharzes; die mit Schwefelsäure übersättigte alkalische Lösung aber klärte sich bald und die ausgeschiedenen bräunlichen Flocken sammelten sich am Boden.

$$
\text { 6. } 11 .
$$

Der Extraktivstoff hatte dieselbe etwas dunklere Farbe, wie der ächten Wurzeln, schmectste eben so, nur weniger 
süfslich und zog wie die andere die Feuchtiglteit der Luft an. Die wässrige Lösung, röthete stark das Lakmuspapier; schwefelsaures Eisenoxydul brachte sogleich eine starke Trübung hervor, nachher entstand ein reichlicher olivengrüner, ins Blauschwarze gehender Niederschlag; Eisenchlorid bewirkte eine olivengrüne Trübung und Niederschlag; Quecksilberchlorid schwache Trübung; neutrales und basisch essigsaures Blei verhielten sich wie bei dem Extraltirstoffe der Jalape, nur waren die Flüssigkeiten mebr entfärbt; eben 80 wirkte das salpetersaure Silber, kleesaure Ammonium und Zinnchloriir; Leimlösung brachte einen minder starken Niederschlag hervor als mit dem Extraktirstoffe der Ip. Purga.

$$
\text { 6. } 12 .
$$

Die anfserordentliche äufsere Aehnlichbeit der officinellen Jalapenwurzel mit der der Ipomoea Purga, verbunden mit dem eben angegebenen fast gleichen chemischen Verhalten ( da wir wohl auf die verschiedene Reaktion der Leimlö. sung, welche in der I. Purga einen geringen Gehalt an Gerbestoff anzeigt, Leinen sehr grofsen Werth legen dürfen) lassen wohl mit Recht anf die Identität beider Wurzeln schliefsen. Es blieb daher nur noch übrig, die allgemeine Einwirkung des Purga-Harzes aut den gesunden menschlichen Organismus zu prüfen. In dieser Absicht nahm einer von uns während einer Stunde 4 Gran des Harzes der Ipom. Purga in zwei Gaben, mit etwas Milchzacher abgerieben. Bald nach dem Einnehmen entstand ein anbaltendes Kratzen im Schlunde, vermehrte Speichelabsonderung and eine allgemeine Bewegung in den Eingeweiden mit gelindem Bauchgrimmen. Nach einer Stunde erfolgte Oeffnung, welches sich bis zum Abend noch einmal wiederholte. - Das Harz der Jalapa spuria wurde einem sebr starken and gesuoden Manne zu 5 Gran auf einmal gegeben, worauf die eben angegebenen Erscheinurgen und drei Stühle erfolgten. Durch 
dieso Wirlung und das chemische Verhalten des Extraltiv. stoffes letzterer Wurzel halten wir uns berechtigt anzuneh. men, dals diese Jalapa spuria ejner der ipumoea Purga nadie verwandren Pflanze angehöre.

$$
\text { \$. } 13 .
$$

Bei dieser Gelegenheit müssen wir auch eines im Handel vorhornmenden und von einem Höllner Hause bezogenen Ja lapenharzes erwä̉nen, das aul den ersten Blich nichts Verdächtiges zu ertennen gibt. Mit Aether sowohl als wit Al. bohol übergossen löst es sich vollständig und in T'erpenthinöl zar Hälfte auf. Eben so war das Verhulten gegen Aetzbalilauge und Salpetersäure, wie das der geistigen und alkalischen Lösung demjenigen des Harzes der Jalapa sparia vö́lig gleich. In einem Löffel erwärmt und später verbransit war nicht der geringste Terpenthingeruch zu bemerlien; auch schmeckte und roch das Harz wie äclites Jalapenharz; sieben Gran desselben bewirkten bei einem gesunden Masne nounmaligen Stublgang.

\section{14.}

Dieses Verhalten belehrte uns zwar, dafs wir es nicht mit einem Harze aus der äcliten Jalapenwurzel zu thun hatton; wabrscheinlich ist dieses Harz aber auch hein lü̈nstliches Gemisch aus Jalapenharz und irgend einem andern harzigen Stoffe, und wir dürfen vielnehr aus den gleichen chemischen Eigenschaften dieses Harzes mit dem aus der Jalapa spuria bereiteten als höchst wahrscheinlich aussprechen, dafs beide Harze aus der Wurzel einer Pflanzenart gewonnen warden. Das häutigere Vorkommen dieser sehr harzreichen (2a in 120) Jalapa spuria im Handel und ihr ais Rad. Jalapae nicht sehr emptehlendes Aeufsere lassen vernuthen, dafs sie schon in den ersten Händen zur Darstellung dieses Handel6artikels benutzt werde, über desseu Werth als Argneimittel weitere ärztliche Beobachtungen entscheiden mögen. 


$$
\text { 6. } 15 .
$$

Wenn wir jetzt noch einmal die Resultate unserer Untersuchung kurz zusanmenfassen wollen, so sind diese folgende: Die Ipomoea Purga Wend. ist die Mutterpflanze der gewïhnlichen officinellen Rad. Jalapae und es wä̀e vielleicht mög. lich; dul's dieselbe in unserm Klima ctrltivirt werden kônnte - Es kommt aber auch eine zweite Sorte Jalapenwurzel in Handel ror, die allem Anscheine nach ron einer weit stärKeren Wurzel abstammt und die nir fär die der Ipomoea Jalapa Desf. halten; es wären so beide Pflanzen als officinell anzunehmen. - Diese zweite Sorte der Jalapa dient endlich wohl sebr häufig zur Bereitung derjenigen Resina Jalapae, welche von Droguisten bereitet wird, und die, obgleich im Verhálten gegen Aether verschieden, doch als Resina Jalapae zu betrachten ist.

Um tabrigens die grofse Uebereinstimmung der Wurzel der Ipomoea Purga mit der officinellen Jalapenwurzel darzuthun, Haben wir hier noch eine möglichst getreue Abbildung der beiden hiev abgehandelten Wurzeln beigefügt. - Die Figuren 123 stellen die getrockmete Wurzel der in Kassel cultivirten Ipomoea Purga $W$. dar. Fig. $4-4$ ist unsere falsche Jalapen - Wurzel. 